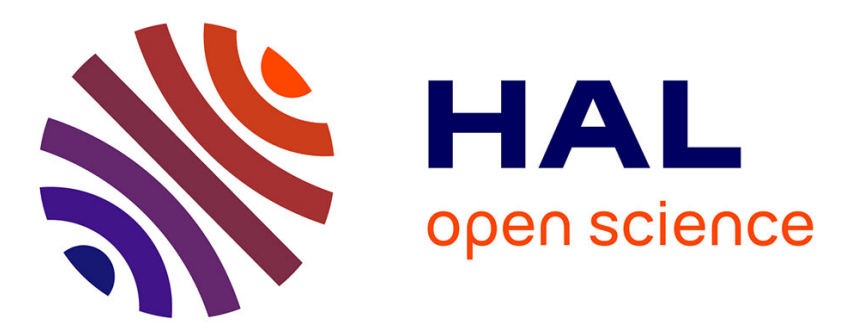

\title{
Effect of a Surfactant Mixture on Coalescence Occurring in Concentrated Emulsions: The Hole Nucleation Theory Revisited
}

\author{
Huy-Hong-Quan Dinh, Enric Santanach-Carreras, Marie Lalanne-Aulet, \\ Véronique Schmitt, Pascal Panizza, François Lequeux
}

\section{To cite this version:}

Huy-Hong-Quan Dinh, Enric Santanach-Carreras, Marie Lalanne-Aulet, Véronique Schmitt, Pascal Panizza, et al.. Effect of a Surfactant Mixture on Coalescence Occurring in Concentrated Emulsions: The Hole Nucleation Theory Revisited. Langmuir, 2021, 37 (29), pp.8726-8737. 10.1021/acs.langmuir.1c00975 . hal-03292845

\section{HAL Id: hal-03292845 \\ https://hal.science/hal-03292845}

Submitted on 20 Jul 2021

HAL is a multi-disciplinary open access archive for the deposit and dissemination of scientific research documents, whether they are published or not. The documents may come from teaching and research institutions in France or abroad, or from public or private research centers.
L'archive ouverte pluridisciplinaire HAL, est destinée au dépôt et à la diffusion de documents scientifiques de niveau recherche, publiés ou non, émanant des établissements d'enseignement et de recherche français ou étrangers, des laboratoires publics ou privés. 


\title{
Effect of a Surfactant Mixture on Coalescence Occurring in Concentrated Emulsions: The Hole Nucleation Theory Revisited
}

\author{
Huy-Hong-Quan Dinh,* Enric Santanach-Carreras, Marie Lalanne-Aulet, Véronique Schmitt, \\ Pascal Panizza, and François Lequeux
}

Cite This: https://doi.org/10.1021/acs.langmuir.1c00975

Read Online

ACCESS |

山ll Metrics \& More

回国 Article Recommendations

SI Supporting Information

ABSTRACT: By conducting both a bottle test and isolate drop-drop experiments, we determine the coalescence rates of water droplets within water-in-oil emulsions stabilized by a large amount of Span 80 in the presence of Tween 20, a surfactant that acts as a demulsifier. Using a microscopic model based on a theory of hole nucleation, we establish an analytical formula that quantitatively predicts the coalescence frequency per unit area of droplets whose interfaces are fully covered by surfactant molecules. Despite its simplicity and the strong assumptions made for its derivation, this formula captures our experimental findings on Span 80stabilized emulsions as well as other results, found in the literature, remarkably well on a wide range of water-in-crude oil systems.

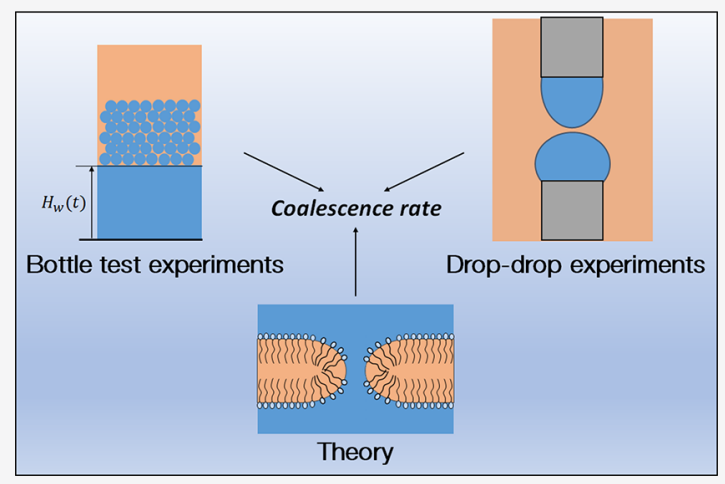

\section{INTRODUCTION}

Water and oil do not mix when whisked together. The mechanical energy input enables the system to form an emulsion state that is a dispersion of water droplets in oil or oil droplets in water. However, this state per se is unstable because of the natural tendency for water and oil to phase separate in order to reduce their interfacial area and hence their interfacial energy. ${ }^{1}$ If the mixture consists only of oil and water, then the duration of such a phase separation process is typically on the order of a few seconds. However, the presence of surfactant molecules in the emulsion strongly enhances its stability. The characteristic time scales for the destabilization process can then span a wide range from a few minutes up to several months or years depending on the physicochemical characteristics of the system. During this destruction process, the average droplet size tends to increase with time through several possible mechanisms such as Ostwald ripening or coalescence. ${ }^{1}$ Coalescence which is the fusion of two adjacent droplets by the rupturing of the thin film that separates them is thus an essential mechanism that controls emulsion stability. The adsorption of surfactant molecules at water-oil interfaces is known to improve the stability of emulsions against coalescence. However, hitherto the underlying physics of coalescence has remained elusive and has still been a subject of debate in the scientific community.

Several scenarios for coalescence have been proposed in the literature. For concentrated emulsion systems having high amounts of surfactant, the thickness of the interstitial thin film between two droplets fully covered by surfactant molecules can reach the size of a molecule. ${ }^{2}$ In such a regime under which the rupture of a thin film is governed by thermal fluctuations, ${ }^{3-5}$ coalescence is expected to be a stochastic phenomenon with a probability for the fusion of two droplets that increases with their contact area. $^{6-9}$ This coalescence scenario has recently been experimentally confirmed in $2 \mathrm{D}$ foams. ${ }^{10}$ The coalescence rate per unit contact area, $\omega$, is therefore a key parameter for emulsion science because it determines the stability of such emulsion systems. Recently, Dinh et al. ${ }^{11}$ have shown that the value of this parameter can easily be extracted from bottle test experiments performed on concentrated emulsions.

Kabalnov et al. ${ }^{12,13}$ have elucidated the crucial role played by the spontaneous curvature of the surfactant films in selecting the nature of the emulsions that naturally form when water and oil are mixed together. Surfactant films with negative (respectively positive) spontaneous curvatures tend to stabilize $\mathrm{W} / \mathrm{O}$ (respectively $\mathrm{O} / \mathrm{W}$ ) emulsions. According to these authors, the coalescence rate per unit area, $\omega$, also strongly depends on the spontaneous curvature of a surfactant monolayer since this parameter affects the bending energy cost required to nucleate a transient neck between two droplets. Although the approach of Kabalnov et al. ${ }^{12}$ well anticipates the nature of an emulsion and its stability, it eventually fails to quantitatively predict an emulsion lifetime and to relate it to the spontaneous curvature of the surfactant monolayers present in the system. Several studies

Received: April 9, 2021

Revised: July 2, 2021 

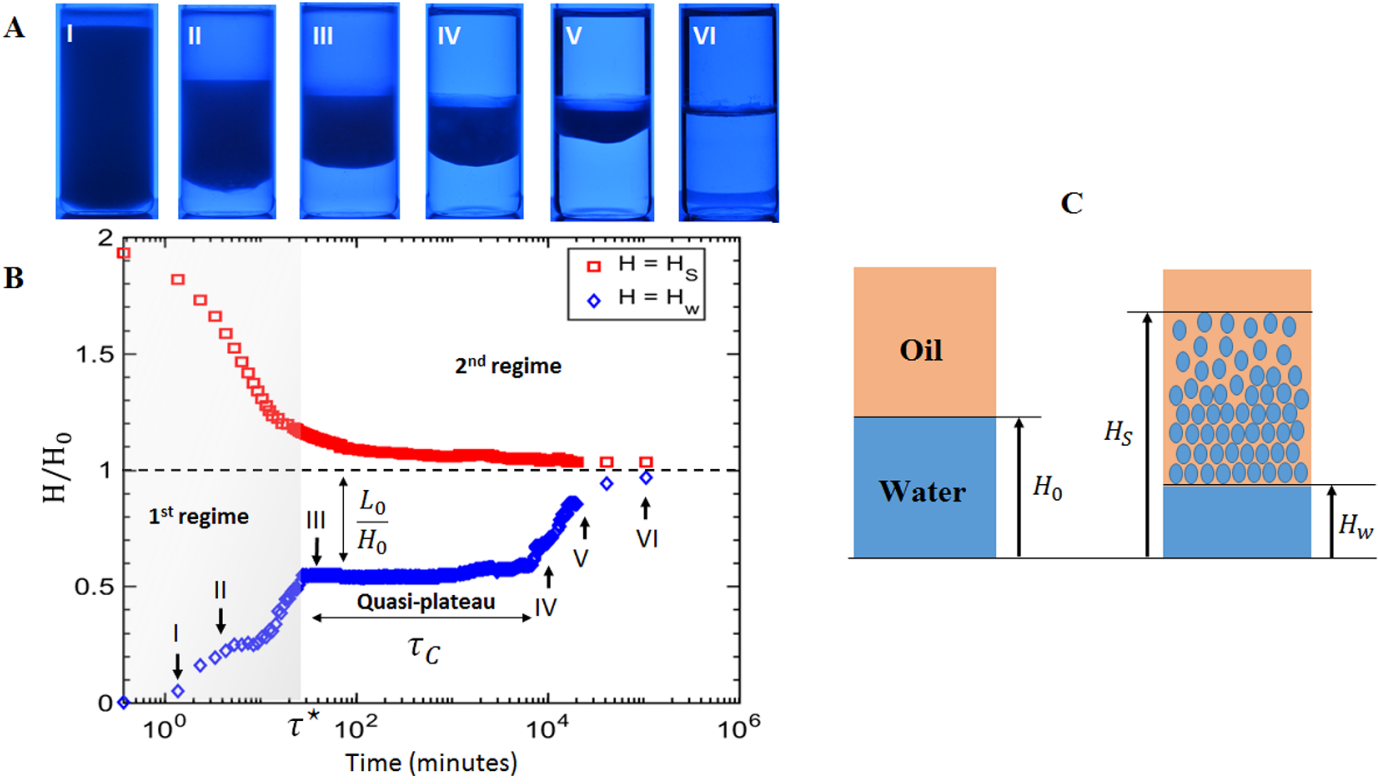

Figure 1. (A) Snapshot images of the destruction kinetics process observed in a bottle test experiment. The emulsion is formed by mixing a dodecane phase containing $800 \mathrm{ppm}$ Span 80 and a water phase containing $10 \mathrm{mM} \mathrm{NaCl}$. (B) Time evolution of the positions of the two interfaces $H_{\mathrm{W}}$ (blue diamonds) and $H_{S}$ (red squares) that are detected in the experiment reported in panel A. (C) Schematic representation of the two macroscopic interfaces that form during the destruction of a W/O emulsion in bottle test experiments. Definitions of $H_{\mathrm{W}}$ and $H_{\mathrm{S}}$, the respective positions of the water/emulsion and emulsion/oil interfaces from the bottom of the vial, and of parameters $\tau_{\mathcal{c}} \tau^{\star}$, and $L_{0}$, discussed in the main text of the article. Figure adapted from ref 11 .

performed on $\mathrm{O} / \mathrm{W}$ and $\mathrm{W} / \mathrm{O}$ emulsions obtained from Winsor I and Winsor II systems, respectively, illustrate this fact very well. A possible explanation for this may lie in the fact that an emulsion lifetime depends not only on the coalescence frequency but also on the average droplet size. ${ }^{11}$ During the emulsification process, the average droplet size, which results from a mechanical balance between hydrodynamic and interfacial forces, is monitored by the value of the interfacial tension (IFT), a parameter that can be related at a molecular scale to the shape of a surfactant molecule and therefore to the spontaneous curvature of the monolayer it forms at interfaces.

In the present study, we extend the approach used by Dinh et al. $^{11}$ to now investigate the coalescence of concentrated emulsions prepared with a binary surfactant mixture, whose surfactant molecules present opposite spontaneous curvature of their monolayers. We present experimental and theoretical evidence that water-soluble Tween 20 acts toward W/O emulsions, stabilized by Span 80 , as a demulsifier that may considerably reduce the emulsion lifetime. Following the methodology recently developed by Dinh et al., ${ }^{11}$ we determine the value of $\omega$ for such emulsion systems from bottle test measurements. We then systematically study the variation of this parameter with the composition of the surfactant mixture used to prepare the emulsion. Our experimental findings agree well with values obtained from drop-drop experiments. Using a theoretical framework built on the thermodynamics of hole nucleation, ${ }^{12}$ we then present a microscopic model that well describes coalescence occurring in concentrated emulsions. We derive an analytical expression for $\omega$, the coalescence rate per unit of area, in terms of the water/oil interfacial tension and the physicochemical parameters of the emulsion system at play in the problem. We demonstrate the robustness and universality of our model by comparing its predictions with experimental findings found in the literature for a wide range of water-in-crude oil emulsion systems.

\section{MATERIALS AND METHODS}

2.1. Emulsion Preparation and Bottle Test Experiments. The continuous phase of the $\mathrm{W} / \mathrm{O}$ emulsions studied herein consists of dodecane (Thermo Fisher Scientific ref. 117590025) to which we add $800 \mathrm{ppm}\left(\sim 8 \times \mathrm{CMC}, 1 \mathrm{ppm}=1.8 \times 10^{-3} \mathrm{~mol} / \mathrm{L}\right)$ Span 80 (Fisher Scientific ref.15474919), a surfactant that favors the formation of W/O emulsions. For the aqueous dispersed phase, sodium chloride (Fisher Scientific) is added to deionized water $(18 \mathrm{M} \Omega)$. The role of $\mathrm{NaCl}$ is twofold because it screens out all possible electrostatic interactions arising from residual traces of ionic surfactant molecules ${ }^{14}$ and prevents Ostwald ripening from being the prevalent destabilization mechanism of the emulsion. To ensure the robustness of our results, we carefully perform tests working with both 10 and $100 \mathrm{mM} \mathrm{NaCl}$ (Debye length smaller than $3 \mathrm{~nm}$ ), and the experimental results are essentially the same between these two salt concentrations. For all of the bottle tests presented hereinafter, we use $24 \mathrm{~mL}$ cylindrical vials of approximately 2 $\mathrm{cm}$ diameter and we work with a water/oil volume ratio of 1 and a total volume of fluids of $15 \mathrm{~mL}$. We proceed as follows: we first pour into a vial $7.5 \mathrm{~mL}$ of water that we then complete with an addition of $7.5 \mathrm{~mL}$ of oil. As water and dodecane are sparingly miscible with each other, we let these two fluids equilibrate at rest for at least $30 \mathrm{~min}$ before starting the emulsification process. We prepare our emulsions with an Ultra-Turrax (IKA-T10) working with a rotational speed of $15000 \mathrm{rpm}$. Under these operating conditions, the mean droplet size is in the range of 7-12 $\mu \mathrm{m}$.

As witnessed in Figure 1, because of the density difference between water and oil phases, gravity-driven settling of water droplets generates over time two noticeable macroscopic interfaces in the system: a water/ emulsion interface observed at the bottom of the vial which creates a water leg and an emulsion/oil interface found at the top of the same vial. We quantify the time evolution of emulsions and characterize their stability by following the positions of these two interfaces over time. We name their respective positions $H_{\mathrm{W}}$ and $H_{\mathrm{S}}$, taken from the bottom of the vial, as depicted in Figure $1 \mathrm{~b}$. To capture $H_{\mathrm{W}}$ and $H_{\mathrm{S}}$ at different stages of the destabilization process, we image the emulsion system at evenly spaced time intervals using a Nikon D5100 camera working with backlighting (Figure 1a).

From each image taken, we extract the positions of both the water/ emulsion $\left(H_{\mathrm{W}}\right)$ and emulsion/oil $\left(H_{\mathrm{S}}\right)$ interfaces using custom-written MATLAB image processing software. Note that since these two 
interfaces do not necessarily appear as horizontal straight lines, some significant uncertainties in the experimental determination of their positions may result. This is particularly true for the $H_{\mathrm{W}}$ interface, where a meniscus is clearly noticeable (Figure $1 \mathrm{a}$ ). For this reason, we average the intensity of the image along each horizontal line and study the profile $I(z)$ of this averaged intensity along $z$, the vertical direction of the vial height. From this intensity profile, we extract the positions of the two interfaces using a standard intensity gradient method. Note that the noise observed for the $H_{S}$ interface is typically of the order of one droplet radius.

2.2. Interfacial Tension Measurements. The formation and stability of an emulsion greatly depend on the amount and nature of surfactant molecules present in the system. Their adsorption on the oil/ water interface also lower the value of the water/oil interfacial tension, a parameter that plays a critical role in the selection of the droplet size during the emulsification process. For this reason, we well characterize the water/oil interfacial tension, $\gamma$, as a function of the concentration of the surfactant that is systematically varied in our model emulsion systems. We measure $\gamma$ in these systems with a pendant drop tensiometer (Tracker, from Teclis-Scientific, France). Briefly, a drop of water of typically a few microliters immersed in the oil phase is formed at the bottom tip of a vertical needle with a syringe. The temperature is $23^{\circ} \mathrm{C}$. The drop profile is determined by image analysis, from which the surface tension is derived. Under mechanical equilibrium of capillary and gravity forces, the Laplace equation relates the pressure difference across the interface, the surface tension, and the surface curvature. Note that before performing any measurement, water and oil phases are left in contact for at least $24 \mathrm{~h}$ so that the partitioning of the surfactant between both phases can reach its equilibrium state. For all of our systems, by systematically varying the concentration of the surfactant in the bulk, we can deduce the equilibrium isotherm adsorption curve which is of primary importance for our modeling work by using the Gibbs adsorption equation and our measurements of interfacial tension. The data are shown in the SI. The CMCs of Span 80 and Tween 20 are roughly $100 \mathrm{ppm}$.

2.3. Drop-Drop Experiments. An effective method for studying the rate of coalescence at the drop scale is the drop-drop experiment. Such an experiment consists of contacting two facing drops and measuring their drop rest time, which is the time elapsed between their contact and their merging. ${ }^{15}$ In our study, two water drops are formed at the tips of two vertical needles facing each other and immersed in an oilfilled tank, as depicted and defined in Figure 2. The volume of the two drops which is typically on the order of $2 \mu \mathrm{L}$ is finely tuned by means of a syringe driver. Once formed, these two drops are allowed to rest, for a certain duration, called the aging time. This duration (typically $5 \mathrm{~min}$ in our experiments) is chosen to be large enough that the adsorption of the surfactant molecules at the interface, which occurs by diffusion from the bulk, can be completed and reach its equilibrium state. This diffusion time can be estimated from the interfacial tension measurements (SI). After this equilibration time, the upper needle which is mounted on a vertical linear motor stage connected to a controller is slowly translated downward until the drop attached to its tip meets the other motionless drop. The two drops are then left at rest until they merge. A side-view video of the system recorded with a CCD camera, working at $1 \mathrm{fps}$, allows one to determine the drop rest (or coalescence) time. Because of its important weight that overcomes capillary forces, the large drop that forms after the merging of the two drops detaches from the tip of the bottom needle and directly falls to the bottom of the tank. The upper needle is then translated up to its initial position, two new drops are created at each tip of the two needles, and another experiment is conducted. This automatic setup which allows one to perform highthroughput measurements under the same experimental conditions is a useful tool for the statistical study of coalescence times. All of our experimental data are collected over approximately 50 droplet pairs, working at an operating temperature of $23{ }^{\circ} \mathrm{C}$.

\section{EXPERIMENTAL RESULTS}

To investigate and characterize coalescence occurring in concentrated emulsions, we perform both drop-drop experiments and bottle test (a)

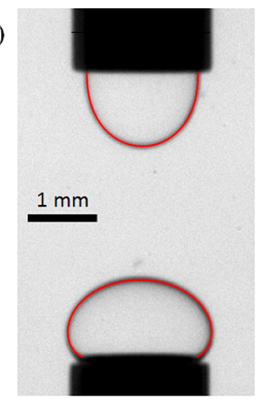

(b)

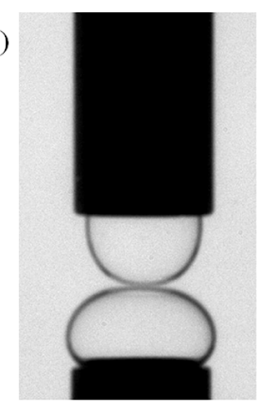

Figure 2. Side-view images of the drop-drop experiment used to investigate the coalescence between two water drops immersed in dodecane (a) before and (b) after the two drops are in contact. In (a), the continuous red lines correspond to the best shape profiles of the drops numerically found by balancing the surface tension and gravitation. The volumes of the upper and lower drops determined through image analysis are respectively 1.6 and $2.9 \mu \mathrm{L}$. The corresponding Bond numbers are 0.55 and 4.4, respectively. In our experiment, the Bond number is defined as $B_{\mathrm{o}}=\frac{\Delta \rho g R_{0}^{2}}{\gamma}$, where $\Delta \rho$ and $\gamma$ are respectively the density difference and the surface tension between the two phases and $g$ and $R_{0}$ are the gravity acceleration constant and the curvature radius at the apex of the drop. The surface tension that is numerically obtained by image analysis is $5.2 \mathrm{mN} \mathrm{m}^{-1}$.

experiments. With these two techniques, we study the destabilization through coalescence of $\mathrm{W} / \mathrm{O}$ emulsion systems stabilized by $800 \mathrm{ppm}$ Span 80 to which we add various amounts of Tween 20 while maintaining the volume ratio of water over oil at 1. Span 80 (HLB 4.3) and Tween 20 (HLB 16.7) are two surfactant systems that are well known to promote the formation of $\mathrm{W} / \mathrm{O}$ and $\mathrm{O} / \mathrm{W}$ emulsions, respectively. Because of their different packing parameters, ${ }^{16,17}$ Tween 20 molecules form interfacial films having the opposite spontaneous curvature to that formed with Span 80 molecules. This water-soluble surfactant which naturally tends to form $\mathrm{O} / \mathrm{W}$ emulsions should therefore act with respect to the reference $\mathrm{W} / \mathrm{O}$ emulsion system as a demulsifier. In order to investigate this effect, we characterize the coalescence rate per unit area, $\omega$, of these various $\mathrm{W} / \mathrm{O}$ emulsions and study how its variation correlates to the amount of Tween 20 added to the system.

3.1. Measurement of Coalescence Frequencies Using Bottle Test Experiments. In bottle test experiments, the phase separation and destruction of $\mathrm{W} / \mathrm{O}$ emulsions stabilized by a large numbers of surfactant molecules occur in a complex but reproducible manner. As illustrated in Figure 1a, this process involves two macroscopic interfaces: a water/emulsion and an emulsion/oil interface. Their respective positions, $H_{\mathrm{S}}$ and $H_{\mathrm{W}}$, defined as in Figure $1 \mathrm{~b}$, evolve over time. In the first regime, typically lasting a few tens of minutes, the water droplets settle because of the density difference between water and dodecane. As a result, a noticeable oil/emulsion interface forms and its position in the vial, $H_{\mathrm{S}}$, decreases over time, until the volume fraction of the dispersed phase reaches a high value of typically $0.8-0.9$ and the droplets become closely packed. This dense emulsion is then compressed at a much lower rate via the drainage of oil through the interstitial films that separate water droplets so that $H_{S}$ decreases much more slowly over time than initially, as revealed by Figure 1c. During the early stages of the first settling regime, since water-oil interfaces are still unsaturated with surfactant molecules, water droplets may easily merge because of coalescence. ${ }^{18}$ The larger and heavier drops that form upon the fusion of two droplets settle faster to the bottom of the tank than the smaller initial droplets. These large drops become unstable when reaching the bottom of the vial and create over time a water leg whose position, $H_{\mathrm{W}}$, increases with time before reaching, at $t=\tau^{*}$ typically on the order of $1-10^{2}$ minutes, a quasi-steady-state position $H_{\mathrm{W}}^{\star}$ (Figure $1 \mathrm{~b}$ ). For $t>\tau^{*}$, a second regime, referred to as the dense emulsion (or quasi-plateau) regime, takes place. In this regime, the height of the dense emulsion zone $L=H_{\mathrm{S}}-H_{\mathrm{W}}$ does not evolve significantly over a 
long period of time $\tau_{\mathrm{c}}$, which may last up to $10^{5}$ minutes. After this duration, the phase-separation kinetics accelerates ${ }^{11}$ and a third regime is witnessed. As shown in Figure 1c, in the dense emulsion regime, the position of the water/emulsion interface, $H_{\mathrm{W}}$, increases very slowly with time, indicating that coalescence events still take place in the system, but at a much slower rate than in the first settling regime. This results from two combined effects. First, the surfactant molecules had enough time to adsorb efficiently at the water-oil interfaces so that an equilibrium between the surface concentration $(\Gamma)$ and the concentration of surfactant molecules present in the bulk can be reached. Second, as a result of coalescence events occurring in the first regime, the oil-water total area has adequately decreased over time so that there are now sufficiently available surfactant molecules in the system to ensure a high surface concentration coverage of the oil-water interfaces. By considering that the minimum surface concentration $\Gamma^{\star}$ required to significantly impede coalescence events is roughly $80 \%$ of the saturation value, $\Gamma_{\text {sat, }}$ Dinh et al. ${ }^{11}$ have demonstrated that the initial thickness, $L_{0}$, of the dense emulsion zone, observed at $t=\tau^{*}$, then varies accordingly to

$$
\frac{L_{0}}{H_{0}}=\frac{R_{0}}{3 \Gamma^{\star}}\left(c_{0}-c^{\star}\right)
$$

with $H_{0}$ being the initial height of the water column in the vial prior to emulsification, $R_{0}$ being the mean droplet radius reached at the start of the dense regime, $c_{0}$ being the concentration of surfactant molecules in the system, and $c^{\star}$ being the minimum surfactant concentration required to generate the dense zone. In the emulsion model system prepared with Span 80 and no Tween $20, c^{\star} \simeq 20$ ppm. It is worthwhile to note that eq 1 predicts that for emulsion systems with similar dispersed-phase volume fractions and average droplet sizes, the initial height of the $\mathrm{W} / \mathrm{O}$ emulsion dense zone that can be witnessed linearly increases with $c_{0}$, the concentration of surfactant used to prepare the emulsion.

In the emulsion dense zone, as the droplet surfaces are nearly saturated with surfactant molecules, coalescence is a very slow kinetic process that is thermally activated. A key parameter in characterizing this phenomenon is therefore the coalescence rate per unit area, $\omega$. The quasi-plateau which lasts for a period of time, $\tau_{\mathcal{c}}$ is followed by a third regime where the phase-separation kinetics accelerates, as depicted in Figure 1. Dinh et al. ${ }^{11}$ have proposed a microscopic model that well explains these various macroscopic observations. They assume that coalescence is the main factor for the destabilization of the emulsion and that $\omega$ remains constant during the whole destruction process. Within the dense zone, they show that the contact area between two closed compact droplets increases linearly with depth $z$ because of the felt hydrostatic pressure that varies as $\Delta P \simeq \Delta \rho g z$, so their probability of coalescence also increases linearly with $z$. As a result, droplets close to the water interface grow faster and are usually larger than upper ones. On the basis of these ingredients, they develop a kinetic model that describes the time evolution of the average droplet size, $R(z, t)$. The variation over time of $R(z, t)$ that is predicted is very sharp and diverges at a finite time $\tau(z)$ which increases with $z$. By taking for the duration of the dense emulsion regime $\tau_{\mathcal{O}}$ the shorter divergence time of the system which corresponds to that of the droplets in contact with the water/ emulsion interface column (i.e., $z \simeq L_{0}$ ), they derive the following analytical expression for the duration of the dense regime, $\tau_{\mathcal{c}}$, in terms of the relevant physical parameters at play ${ }^{11}$

$$
\tau_{\mathrm{c}}=\frac{\gamma}{2 \pi \omega \Delta \rho g L_{0} R_{0}{ }^{3}}
$$

where $\gamma, g$, and $\Delta \rho$ are the equilibrium oil-water interfacial tension (IFT) of the system, the acceleration due to gravity and the density difference between the aqueous and oil phases, respectively. When the first transient regime exists (i.e., when $L_{0}<H_{0}$ ), by combining eqs 1 and 2 , one may then establish a simple relationship among $\omega$, the physicochemical parameters characterizing the emulsion system $\left(c_{0}, c^{*}\right.$, $\Gamma_{\text {sat }}$, and $\left.\Delta \rho\right)$, and two easily measurable macroscopic quantities $\left(\tau_{\mathrm{c}}\right.$ and $\left.L_{0}\right)$ characterizing the dense emulsion regime occurring in the bottle test experiments, as shown below: ${ }^{11}$

$$
\omega=\frac{\gamma}{27 \pi \tau_{\mathrm{c}} \Delta \rho g H_{0}}\left(\frac{c_{0}-c^{\star}}{\Gamma_{\text {sat }}}\right)^{3}\left(\frac{L_{0}}{H_{0}}\right)^{-4}
$$

Equation 3 is a promising relationship that offers a simple and inexpensive method to determine values of $\omega$ from bottle test experiments. Herein, we decided to apply this methodology to study the stability of Span 80-stabilized W/O emulsions, when Tween 20, another surfactant well known to favor the formation of $\mathrm{O} / \mathrm{W}$ emulsions, is added to the system. To do so, we perform systematic bottle test experiments by varying the concentration of Tween 20 that is added to a reference W/O emulsion that is stabilized by $800 \mathrm{ppm}$ Span 80 with $10 \mathrm{mM} \mathrm{NaCl}$ in the aqueous phase and whose water/oil volume ratio is 1 . All experiments are performed at $T=23{ }^{\circ} \mathrm{C}$. As shown in Figure 3, we witness that $\tau_{\mathrm{c}}$ decreases with the concentration of Tween

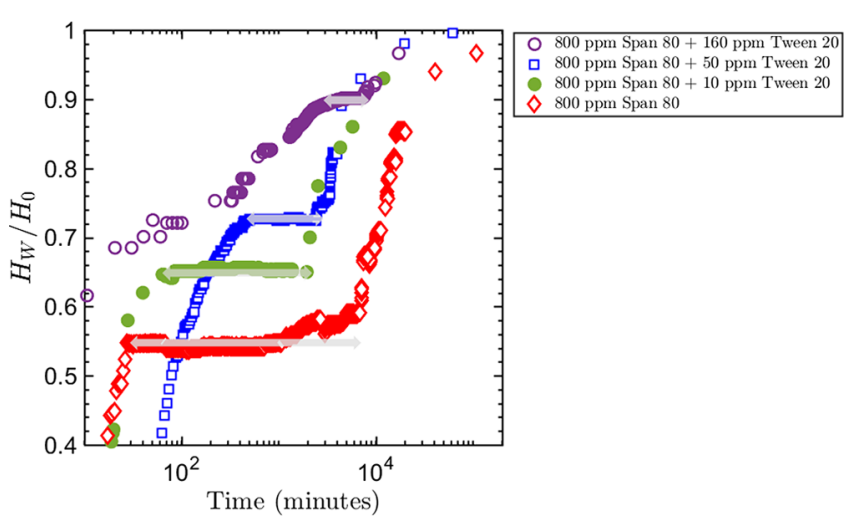

Figure 3. Kinetics of the emulsion destruction process occurring for different $\mathrm{W} / \mathrm{O}$ emulsion systems in a bottle test experiment. All emulsions are stabilized by $800 \mathrm{ppm}$ Span 80 , and they differ from each other only by their respective amounts of Tween 20 . The volume ratio of the water phase over the oil phase is 1 for all of the emulsions, and the vials used to perform these experiments are identical. The oil phase is dodecane, and the water phase consists of $10 \mathrm{mM} \mathrm{NaCl}$ added to deionized water. Note that the smaller the initial droplet size, the longer the first settling regime lasts.

20, a result that clearly indicates that the addition of Tween 20 molecules to the emulsion system is strongly detrimental to its stability. From the experimental set of data $\left(L_{0}, \tau_{c}\right)$ determined from bottle test experiments, we extract the values of $\omega$ by using eq 3 . Since $c^{\star}$ is the minimum concentration of Span 80 that allows the generation of the dense emulsion zone, it can be simply measured for the systems in the presence of Tween 20. For 10, 50, and $160 \mathrm{ppm}$ Tween 20, the values taken by $c^{\star}$ are 60,200 , and $400 \mathrm{ppm}$, respectively. Note that $c^{\star}$ increases with the concentration of Tween 20 . Indeed, since Tween 20 molecules favor the formation of $\mathrm{O} / \mathrm{W}$ emulsions instead of $\mathrm{W} / \mathrm{O}$ emulsions, higher concentrations of Span 80 are needed to compensate for the effect of Tween 20 molecules on the spontaneous curvature of the water-oil interfaces. In Table 1 , we report $\omega$ values that we have found for systems prepared with different concentrations of Tween 20 . For 10 and $50 \mathrm{ppm}$ Tween 20, the values of $\omega$ are $1.2 \times 10^{6}$ and $6 \times 10^{6}$ $\left(\mathrm{s}^{-1} \mathrm{~m}^{-2}\right)$, respectively. The presence of a small amount of Tween 20 such as $10 \mathrm{ppm}$ therefore considerably increases the coalescence frequency per unit area by about a factor of 30 , which is very detrimental to the stability of the $\mathrm{W} / \mathrm{O}$ emulsion.

3.2. Drop-Drop Experiment Validation. To validate our bottle test experimental methodology, we next compare $\omega$ values extracted from bottle test experiments with those measured at the drop scale with drop-drop experiments. Figure $4 \mathrm{a}$ reports the results of drop-drop experiments performed on systems with different concentrations of Tween 20 under similar experimental conditions (i.e., the volumes of the two drops are identical and the temperature is constant, $T=23^{\circ} \mathrm{C}$ ). For each system, the mean coalescence time of the two drops is statistically determined over a total sampling of 50 consecutive dropdrop experiments. As evident from Figure $4 \mathrm{a}$, in the presence of Tween 
Table 1. For the Different Systems Studied, Their Surfactant Mixture Composition, Experimental Data $L_{0}$ and $\tau_{\mathrm{c}}$ Extracted from Bottle Test Measurements, Their Interfacial Tension $\gamma$ Values Measured Using a Commercial Tensiometer (TECLIS), and Their $\omega$ Values Predicted by Equation 3

\begin{tabular}{|c|c|c|c|c|c|}
\hline surfactant mixture & $L_{0} / H_{0}$ & $\tau_{\mathrm{c}}(\min )$ & $\gamma\left(\mathrm{mN} \mathrm{m}^{-1}\right)$ & $c^{\star}(\mathrm{ppm})$ & $\omega\left(\mathrm{s}^{-1} \mathrm{~m}^{-2}\right)$ \\
\hline 800 ppm Span 80 & 0.50 & $8.8 \times 10^{3}$ & 5.2 & 20 & $2.0 \times 10^{5}$ \\
\hline 800 ppm Span $80+10$ ppm Tween 20 & 0.34 & $1.9 \times 10^{3}$ & 4.1 & 60 & $1.2 \times 10^{6}$ \\
\hline 800 ppm Span $80+50$ ppm Tween 20 & 0.25 & $1.8 \times 10^{3}$ & 3.2 & 200 & $6.1 \times 10^{6}$ \\
\hline 800 ppm Span $80+70$ ppm Tween 20 & 0.15 & $3.0 \times 10^{3}$ & 2.5 & 250 & $1.9 \times 10^{7}$ \\
\hline 800 ppm Span $80+120$ ppm Tween 20 & 0.05 & $8.0 \times 10^{3}$ & 1.5 & 380 & $2.0 \times 10^{8}$ \\
\hline 800 ppm Span $80+160$ ppm Tween 20 & 0.10 & $1.8 \times 10^{3}$ & 0.8 & 400 & $3.2 \times 10^{7}$ \\
\hline
\end{tabular}

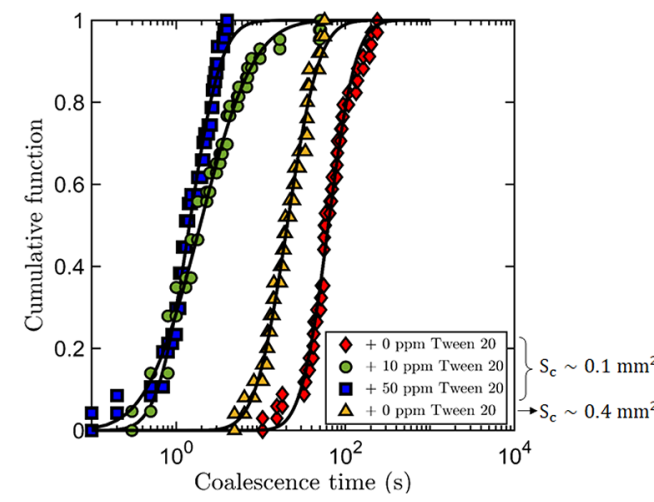

(a)

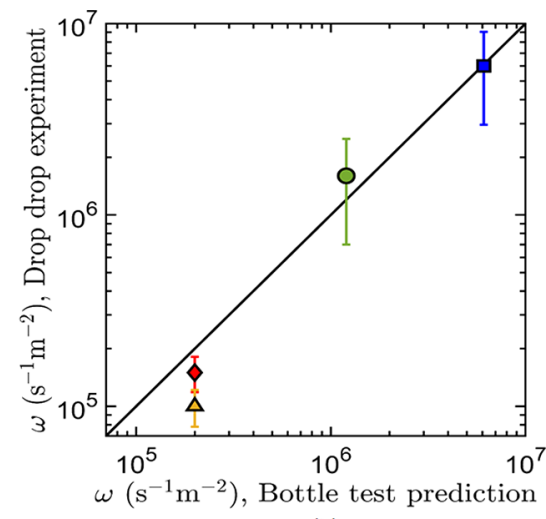

(b)

Figure 4. (a) Cumulative probability distribution of drop rest times at the oil-water interface. Continuous lines indicate the best fits of the data using a log-normal distribution function. (b) Comparison between the values of the coalescence rate per unit area, $\omega$, measured with drop-drop experiments and extracted from bottle test experiments by using eq 3 . The points are the mean values, and the error bars correspond to the standard errors of the experimental data obtained from drop-drop experiments.

20 , the coalescence time of the emulsion system is significantly reduced. From these collected data, we can estimate $\omega$ values under the assumption that the mean coalescence time corresponds to $1 / \omega S_{\mathcal{c}}$ where $S_{\mathrm{c}}$ is the contact area between the two drops. The contact area, $S_{\mathrm{c}}$ is determined through an image analysis of a side view of the two drops at contact, as detailed in Appendix A. As clearly shown in Figure 4b, $\omega$ values measured with drop-drop experiments correlate well with those extracted from bottle test experiments (Table 1). In addition and to ensure our assumption that the coalescence time is inversely proportional to the surface contact, we have performed an additional drop-drop experiment for the system of $800 \mathrm{ppm}$ Span 80 at higher surface contact $S_{\mathrm{c}}$ (Appendix A and Figure 11). The mean coalescence time in this case is indeed lower than the one previously shown (Figure 4a). The obtained coalescence frequency is the same order of magnitude as the obtained value in the other experiment at a lower contact surface.

\section{THEORETICAL INTERPRETATION}

Our experimental results demonstrate that bottle test experiments offer a simple, robust, and inexpensive way of determining coalescence rates in concentrated emulsion systems. Using both this methodology and standard drop-drop experiments, we have shown that the presence of a very small amount of Tween 20 in our $\mathrm{W} / \mathrm{O}$ emulsion system is very detrimental to its stability since it significantly increases the coalescence rate.

This destabilization effect likely results from the competitive adsorption of Span 80 and Tween 20 molecules on water-oil interfaces and the opposite spontaneous curvatures of their respective monolayers. Owing to their different packing parameters, ${ }^{16,17}$ oil-soluble Span 80 and water-soluble Tween 20 molecules have a natural tendency to form $\mathrm{W} / \mathrm{O}$ emulsions and $\mathrm{O} / \mathrm{W}$ emulsions, respectively. Because of the small volume ratio between its hydrophilic head and its hydrophobic tail, Span
80 molecules take the shape of inverted truncated cones. In the sole presence of these molecules, coalescence between two water droplets is hindered because of the high energy cost required to create a hole in their oil interstitial film, which results from a strong steric frustration of the Span 80 hydrophobic tails. With Tween 20 molecules, such steric frustration no longer exists so that the creation energy barrier of such a hole is lowered. The presence of these molecules should therefore enhance the probability of coalescence between two water droplets. Indeed, physical phenomena triggered by curvature frustration are reported not only in emulsions but also in a large number of other systems. $^{19}$

We next rationalize these experimental findings by introducing a model that well explains the effect of a surfactant demulsifier on the destabilization of an emulsion occurring through coalescence. This microscopic model adequately predicts the coalescence rate per unit area, $\omega$, as a function of the composition of the surfactant mixture in the emulsion. It is based on the calculation of $W^{\star}$, the energy cost required to nucleate a transient neck between two droplets that, in the end, leads to their fusion. ${ }^{12}$

Although the mechanism of hole nucleation has drawn much interest over the last few decades, it remains elusive. Kashschiev and Exerowa ${ }^{4,20}$ have proposed that the creation of a hole within a surfactant thin film requires the generation of a depletion zone with a lateral size $L$. Although the energy penalty of such a process, $\gamma_{\mathrm{OW}} \pi L^{2} / 4$ ( $\gamma_{\mathrm{OW}}$ is the surface tension between oil and water with no surfactant), is rather large $\left(>100 k_{\mathrm{B}} T\right)$ for usual surfactant molecules, it may be on the order of $k_{\mathrm{B}} T$ if one considers that $L$ takes smaller values in the range of $1-5 \AA$.

However, the creation of this hole in the bilayer is not the limiting step of coalescence. As discussed below, the spreading 

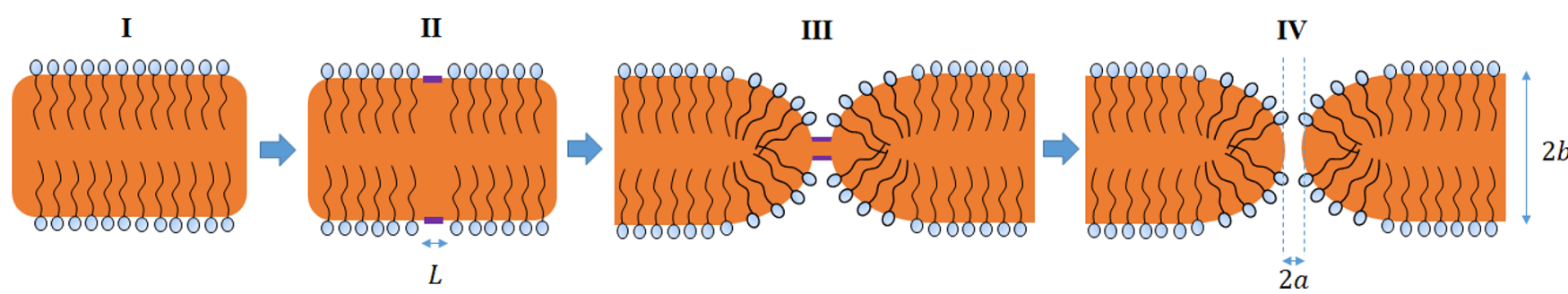

Figure 5. Schematic representation of the nucleation of a hole in a thin surfactant film. A depletion zone with a lateral size on the order of a molecular size is first created in the film (II). Because of attractive van der Waals interactions, the thickness of this depletion zone decreases (III) up to zero when a hole is created and the surfactant film then forms a torus (IV) to minimize its energy.

of the nucleated hole requires another energy barrier to be overcome. If we consider the thermally activated scenario, introduced by Vrij, then the creation of a circular hole within the surfactant film, as depicted in Figure 5, requires a surface energy, given by $^{21}$

$$
W_{\mathrm{S}}=2 \pi \gamma\left[\pi b(a+b)-2 b^{2}-(a+b)^{2}\right]
$$

where $a$ and $b$ are the radius of the hole and the film thickness, respectively. This energy comes from the interplay between the energy penalty at the edge of the hole where the surfactant film is rounded and takes the shape of a semicircular torus and the energy gain in the planar part. When $b$ is constant, $W_{\mathrm{S}}$ takes a maximum value, $2.94 \gamma b^{2}$, for $a \simeq 0.57 b$. This value corresponds to the energy barrier that must be overcome so that the nucleated hole may spread. However, as noticed by Kabalnov et al., ${ }^{12}$ there is also a bending energy penalty to take into account since the monolayer at the edge of the nucleation hole is strongly curved. The relative contribution of this bending term to the total energy may become important when the surface tension is small. The bending modulus of the surfactant monolayer, $\kappa$, which depends on the length of the surfactant tail, ${ }^{22}$ may typically be on the order of a few $k_{\mathrm{B}} T$ for usual surfactant systems. $^{23}$ The relative contributions of the surface and bending terms to the total energy cost are set by the elastocapillary length, $\lambda=\sqrt{\frac{\kappa}{\gamma}}$. When the deformation length of the monolayer is larger (respectively smaller) than $\lambda$, the surface (respectively the bending) energy dominates.

According to Kabalnov et al., ${ }^{12}$ the energy barrier $W(a, b)$ required to open a hole of radius $a$ within a surfactant film of thickness $b$ is the sum of three terms

$$
W(a, b)=W_{\mathrm{S}}+W_{\mathrm{B}}+W_{\mathrm{G}}
$$

where $W_{\mathrm{S}}$ is given by eq $4, W_{\mathrm{B}}$ is the bending energy penalty of the curved film at the edge of the nucleation hole, and $W_{\mathrm{G}}$ is the Gaussian curvature contribution accounting for the fact that the creation of the hole reduces the Euler characteristic of the monoloyer by 2 . As shown by Kabalnov et al., ${ }^{12} W_{\mathrm{B}}$ and $W_{\mathrm{G}}$ are respectively given by

$$
W_{\mathrm{B}}=2 \pi \kappa\left[-2 \pi K_{0} a-2(\pi-4) K_{0} b+\frac{2(a+b)^{2}}{b \sqrt{a(a+2 b)}} a \tan \sqrt{\frac{a+2 b}{a}}-4\right]
$$

and $W_{\mathrm{G}}=-4 \pi \bar{\kappa}$, where $\bar{\kappa}$ and $K_{0}$ are the saddle-splay modulus and the spontaneous curvature of the surfactant monolayer, respectively. Note that, as we will show in the following text, close to the range value of hole radius $a$ at the maximum energy barrier, the three terms $\left(W_{\mathrm{B}}, W_{\mathrm{S}}\right.$, and $\left.W_{\mathrm{G}}\right)$ are of the same order of magnitude and are not significantly different from each other.
Since the monolayer is fully covered by surfactant, the thickness of the thin film is extremely small and corresponds to a Newton black film. ${ }^{2,24}$ We therefore claim that $b$ must be as small as possible and therefore takes a value $b_{0}$ which is about half the thickness of a surfactant bilayer. The energy barrier $W^{\star}$ that has to be overcome for the hole to spread then corresponds to the maximum of the function $W\left(a, b_{0}\right)$ versus $a$, which can be found numerically. A rapid dimensional analysis of the problem reveals that $W^{\star}$ can be expressed as a function of $\gamma b_{0}^{2}$ and three dimensionless parameters of the system, namely, $\frac{b_{0}}{\lambda}, K_{0} \lambda$, and $\frac{\bar{\kappa}}{\kappa}$. However, since the spontaneous curvature $K_{0}$ is indeed related to the interfacial tension $\gamma$ through $K_{0}=-\sqrt{1+\frac{\bar{\kappa}}{2 \kappa}} \sqrt{\frac{\gamma}{2 \kappa}}$ ,23,25,26 the problem has only two dimensionless parameters: $\frac{b_{0}}{\lambda}$ and $\frac{\bar{\kappa}}{\kappa}$. Note that, because of thermodynamic stability, $2 \kappa+\bar{\kappa}>0$ and $\bar{\kappa}<0 .{ }^{23}$ Thus, the variations of $\kappa$ and $\bar{\kappa}$ are limited and the change in the spontaneous curvature $K_{0}$ is strongly related to the variation of the interfacial tension $\gamma . \kappa$ and $\bar{\kappa}$ are related more to the size of the molecules and can be considered in a first order approximation to be nearly constant when the composition of the mixture of surfactants is varied. Furthermore, we have checked that all of the results below weakly depend on the variation of these bending moduli. For our quaternary system made of Span 80, Tween 20, water, and dodecane, we take $b_{0} \simeq$ $1.7 \mathrm{~nm},{ }^{27,28} \kappa=0.59 k_{\mathrm{B}} T$, and $\bar{\kappa}=-0.58 k_{\mathrm{B}} T$. $^{23}$ The only remaining variable in our problem is therefore the interfacial tension $\gamma$. As detailed in Appendix B, the third term on the righthand side of eq 6 can be replaced by a linear approximation, and we can thus establish an analytical expression that relatively well approximates the energy barrier, $W^{\star}$ :

$$
\begin{aligned}
W^{\star} & \simeq \frac{\pi^{3}}{2} \frac{\left(b_{0}^{2} \gamma+\sqrt{2 \kappa+\bar{\kappa}} b_{0} \sqrt{\gamma}+\frac{\kappa}{2}\right)^{2}}{b_{0}{ }^{2} \gamma}-4 \pi b_{0}{ }^{2} \gamma \\
& -8 \pi \sqrt{2 \kappa+\bar{\kappa}} b_{0} \sqrt{\gamma}-4 \pi(\kappa+\bar{\kappa})
\end{aligned}
$$

As shown in Figure 6, the values of $W^{\star}$ thus determined are very close to those numerically obtained by solving eqs 5 and 6 . For large interfacial tension values, $W^{\star}$ decreases as $\gamma$ decreases since it is mainly governed by the contribution of the surface tension energy term. However, when $\gamma$ reaches sufficiently small values, the contribution of the surface tension energy to $W^{\star}$ may then become smaller than that of the bending energy. In this case, since a large hole must be created in order to compensate for the effect of the bending energy, the value of $W^{\star}$ increases as $\gamma$ decreases.

Under the assumption that coalescence is a thermally activated destruction process, the coalescence rate per unit area then satisfies 


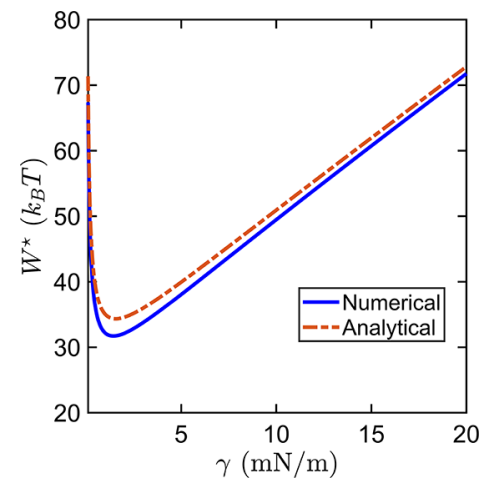

Figure 6. Values of $W^{\star}$ determined by numerically solving eqs 5 and 6 (blue curve) and by using the approximated analytical expression derived in eq 7 as a function of $\gamma$ for $b_{0}=1.7 \mathrm{~nm}, \kappa=0.59 k_{\mathrm{B}} T$, and $\bar{\kappa}=$ $-0.58 k_{\mathrm{B}} T$

$$
\omega=f \exp \left(\frac{-W^{\star}}{k_{\mathrm{B}} T}\right)
$$

where $f$, the characteristic scale of $\omega$, can be estimated roughly as followed. As depicted in Figure 7, the time required for a

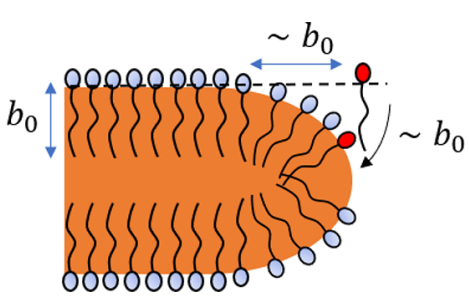

Figure 7. Schematic representation of a surfactant molecule diffusing from the bulk to the curved surface of the surfactant film that forms when a hole is created.

surfactant molecule present in the bulk to diffuse over the characteristic distance $b_{0}$ is $\tau=b_{0}^{2} / D_{s}$, where $D_{s}$ is the diffusion coefficient of surfactant molecules in the bulk. (We consider that the surface diffusion is much slower than diffusion from the bulk.) Now, by taking a characteristic area of $\pi b_{0}^{2}$ which corresponds roughly to that of the smallest hole that can form, the frequency per unit area is $f \approx \tau^{-1} / \pi b_{0}{ }^{2}$, which yields

$$
f \simeq \frac{D_{\mathrm{s}}}{\pi b_{0}^{4}}
$$

From dynamic interfacial tension measurements (SI), we estimate that the diffusion coefficient of Span 80 in dodecane, $D_{s}$, is on the order of $10^{-11} \mathrm{~m}^{2} / \mathrm{s}$, which accounts for the surfactant diffusion and adsorption on the interface. This value is in good agreement with that found in the literature. ${ }^{29}$

By taking $b=1.7 \mathrm{~nm}$ and the obtained value for $D_{\mathrm{s}}$, we first determine the value of $f$ by using eq 9 that we next report in eq 8 . For each surfactant mixture that we have investigated (Table 1), we then compute its energy barrier, $W^{\star}$, by using eq 7 and then deduce its coalescence frequency $\omega$ by using eq 8 . As shown in Figure 8 , very good agreement is found between theoretically predicted values and experimental ones that are reported in Table 1.

Now, if one considers that in eq 8 the contribution of the exponential energy barrier term prevails over that of the prefactor $f$, it sounds acceptable to use in eq 9 the StokesEinstein expression of $D_{\mathrm{s}}$ to estimate $f$. By doing so, we

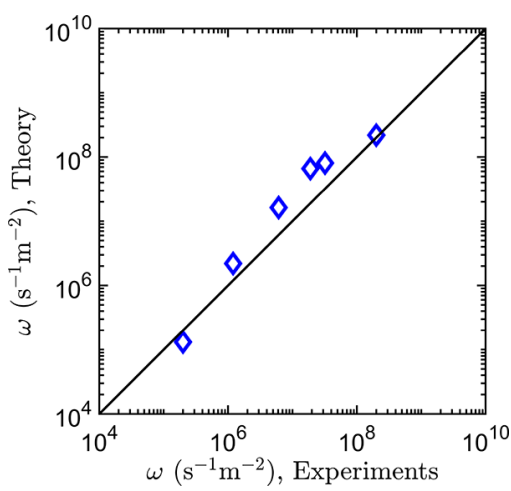

Figure 8. Comparison between experimental and theoretically predicted values of $\omega$ for the various surfactant mixtures investigated in Table 1 . For each system, $\omega$ is predicted by combining eqs $7-9$. The experimental value of $\gamma$ that is reported in Table 1 is inserted into eq 7 .

determine that $f \simeq k_{\mathrm{B}} T / 6 \pi^{2} b_{0}{ }^{5} \eta$, a result that when reported in eq 8 sheds some light on the influence of the viscosity of the continuous phase on $\omega$. Very interestingly, we remark that this relationship well describes the effect of the viscosity of the continuous phase on droplet coalescence that has been qualitatively reported in some experiments. ${ }^{30,31}$

Finally, in the case of very small surface tension, surface fluctuations becomes huge. The consequence is that other phenomena may occur with a cosolubilization of oil and water. In this Winsor III regime, our model no longer applies. Our model is indeed limited to a range of surface tension that corresponds to the Winsor II regime.

In the next section, we will investigate whether this theoretical framework can be used to successfully predict the stability of water-in-crude oil emulsion systems, a very important issue for the petroleum industry.

\section{APPLICATION TO OTHER EMULSION SYSTEMS: TOWARD A UNIVERSAL DESCRIPTION OF THE EFFECT OF A DEMULSIFYING SURFACTANT ON EMULSION STABILITY LOSS}

To explain the increase in the coalescence rate per unit area, $\omega$, that occurs in a concentrated $\mathrm{W} / \mathrm{O}$ emulsion when a surfactant demulsifier is added to it, we have previously introduced a microscopic model. Within this model framework, we have derived several analytical formulas (eqs 7-9) that allow one to predict the value of $\omega$ for a W/O emulsion. By working with Span 80-stabilized W/O emulsions to which a few quantities of Tween 20 (a water-soluble molecule that herein acts as a demulsifier) are added, we have shown that the values of $\omega$ predicted with our model agree very well with those experimentally measured. We now compare the theoretical values of $\omega$ with experimental values for a wide range of other emulsion systems. Our objective is now to investigate whether our theoretical framework can be successfully applied to real life emulsion systems, such as crude oil/additive systems, in order to predict and quantify how their lifetimes are altered by the addition of a demulsifier agent. To illustrate this point, let us first consider, for instance, two W/O emulsion systems $\mathrm{A}$ and $\mathrm{B}$. Their respective continuous and dispersed phases are similar, and their droplets are covered by the same oil-soluble surfactant molecules. However, their temperatures or their compositions (salt contents or concentration of an added demulsifier) may be different. As these various physical parameters are known to affect the spontaneous curvature of a surfactant monolayer, ${ }^{13,32}$ 
the lifetimes of these two emulsions are therefore expected to differ. Since water-in-crude oil emulsions are very complex systems, it is not possible to simply estimate the prefactor $f$ that intervenes in the analytical expression of $\omega$, which we have previously derived. However, it is reasonable to infer that the prefactor, $f$, is the same for systems $A$ and $B$ that differ only from the spontaneous curvatures of their respective surfactant films. Within this assumption, from eq 7 it is straightforward to show that the ratio between their respective coalescence rates $\omega_{\mathrm{B}} / \omega_{\mathrm{A}}$ does not depend on the unknown prefactor, $f$, but only on the difference between their respective energy barriers, $W_{\mathrm{A}}^{\star}$ and $W_{\mathrm{B}}^{\star}$. We next investigate whether this assumption is valid, in other words, if the ratio between the coalescence rates of systems B and $A$ that are experimentally measured agrees with the theoretical prediction obtained with eq 7. This will confirm whether our theoretical framework can be successfully applied to predict the ratio between the coalescence rates of two systems that differ only from their temperature or salt composition or from the addition of additives. If so, then we believe the analytical formula, derived in eq 7 , would offer an unprecedented guide in emulsion science to robustly estimate emulsion lifetimes solely from their IFT values.

To optimize the formulation of emulsions with regard to their stability, bottle tests are widely used in industry as well as in academical research laboratories. The time lapse to reach a certain percentage of macroscopic phase separation is a commonly used variable to characterize an emulsion's stability. For concentrated emulsion systems, we claim here that this quantity is simply proportional to the time duration of their dense emulsion regime, $\tau_{\mathrm{c}}{ }^{11}$ For crude oil emulsions, the concentration of natural emulsifiers present in them is usually very high so that the surface of the droplets is fully covered by these molecules very soon after their creation by emulsification. Consequently, these systems may not exhibit the first regime of separation preceding the dense emulsion (or quasi-plateau) regime that we have previously identified and characterized. ${ }^{11}$ When this occurs, as $L_{0}=H_{0}$, the time duration, $\tau_{\mathrm{c}}$ predicted by eq 3 is given by $\tau_{\mathrm{c}} \approx \gamma / \omega R_{0}{ }^{3}$, ${ }^{11}$ where $R_{0}$ is the initial size of the droplets. For two such emulsions systems A and B, one therefore obtains the following relationship between their respective lifetimes, $\tau_{\mathrm{cA}}$ and $\tau_{\mathrm{cB}}$,

$$
\frac{\tau_{\mathrm{cB}}}{\tau_{\mathrm{cA}}}=\frac{\omega_{\mathrm{A}}}{\omega_{\mathrm{B}}} \frac{\gamma_{\mathrm{B}}}{\gamma_{\mathrm{A}}}\left(\frac{R_{0 \mathrm{~A}}}{R_{0 \mathrm{~B}}}\right)^{3}
$$

where $R_{0 \mathrm{~A}}$ and $R_{0 \mathrm{~B}}$ stand for the initial mean droplet radius of the two systems, respectively.

In most mechanical emulsification techniques, the formation of droplets occurs in turbulent flows. Under such flow conditions, the droplet size results from a balance between interfacial stresses and inertial or viscous stresses. At high Reynolds numbers, turbulent mixing is characterized by a cascade of energy from large to small length scales in the socalled inertial subrange, where inertial stresses prevail over viscous stresses, down to the Kolmogorov length, $l_{\mathrm{K}}$ below which viscosity is predominant in the viscous subrange. ${ }^{33}$ Therefore, depending on whether the size of the droplet is larger or smaller than $l_{\mathrm{K}}$, the interfacial stress is balanced either by the inertial stress or by the viscous stress, respectively. In the inertial subrange, the mean droplet size, $R_{0}$, which results from a balance between the interfacial stress, $2 \gamma / R_{0}$, and the inertial stress, $\rho(\epsilon d)^{2 / 3}$ is therefore given by $R_{0} \approx \gamma^{3 / 5} \rho^{-3 / 5} \epsilon^{-2 / 5}$, where $\rho$ is the density of the continuous phase and $\epsilon$ is the energy dissipation per unit mass and time. ${ }^{34-36}$ The value of the numerical prefactor depends on the design of the emulsifying mixer and on its characteristic length scales. Such a droplet formation regime occurs when the Reynolds number in play is very high. The occurrence of such a regime is promoted by low-viscosity oils used as continuous phases. When the two emulsions, A and B, are generated under similar operating mixing conditions, we can deduce that for the inertial regime of emulsification $\frac{R_{0 \mathrm{~A}}}{R_{0 \mathrm{~B}}}=\left(\frac{\gamma_{\mathrm{A}}}{\gamma_{\mathrm{B}}}\right)^{3 / 5}$. In the viscous subrange, the interfacial stress $\gamma / R_{0}$ is balanced against the viscous stress $\eta \dot{\gamma}$ so that $R$ is then given by $R_{0} \approx \gamma / \eta \dot{\gamma}$, where $\dot{\gamma}$ is the shear rate. We can thus note that in this latter droplet formation regime, $R_{0 \mathrm{~A}} / R_{0 \mathrm{~B}}=\gamma_{\mathrm{A}} /$ $\gamma_{\mathrm{B}} \cdot{ }^{34,37,38}$ Such a regime may experimentally be witnessed when the viscosity of the continuous oil phase becomes large enough. To summarize, when working under the same operating mixing conditions, we note that

$$
\frac{R_{0 \mathrm{~A}}}{R_{0 \mathrm{~B}}}=\left(\frac{\gamma_{\mathrm{A}}}{\gamma_{\mathrm{B}}}\right)^{n}
$$

where the value of the exponent, $n$, is $3 / 5$ for the inertial subrange and 1 for the viscous subrange regimes of droplet formation. These two regimes are experimentally observed by working with low- and high-viscosity oils, respectively. By combining eqs 10 and 11 , it is straightforward to derive that

$$
\ln \left(\frac{\tau_{\mathrm{cB}}}{\tau_{\mathrm{cA}}}\right)=\ln \left(\frac{\omega_{\mathrm{A}}}{\omega_{\mathrm{B}}}\right)+(3 n-1) \ln \left(\frac{\gamma_{\mathrm{A}}}{\gamma_{\mathrm{B}}}\right)
$$

The considered systems A and B differ only from the spontaneous curvature of the surfactant monolayer, and their prefactors $f$ are essentially the same. Therefore, the ratio of the coalescence frequencies $\omega_{\mathrm{A}}$ and $\omega_{\mathrm{B}}$ is

$$
\ln \left(\frac{\omega_{\mathrm{B}}}{\omega_{\mathrm{A}}}\right) \simeq \frac{W_{\mathrm{A}}^{\star}-W_{\mathrm{B}}^{\star}}{k_{\mathrm{B}} T}
$$

where $W_{\mathrm{A}}^{\star}$ and $W_{\mathrm{B}}^{\star}$ are the energy barriers of hole nucleation of systems $A$ and $B$, respectively. By integrating eq 7 into eq 13 , we are able to compute the ratio of coalescence frequencies $\omega_{\mathrm{B}} / \omega_{\mathrm{A}}$ of systems A and B solely from their interfacial tensions. Subsequently, we can substitute into eq 12 to predict the relative stability between two emulsion systems, having both the same aqueous and oil phases, and surfactant monolayers, exhibiting different spontaneous curvature, solely from their water-oil interfacial tensions. This could be of primary interest in the field of emulsion science as it offers a practical way to easily quantify how the stability of an emulsion through coalescence is altered by a change in its temperature, its salinity content, or its composition (if a demulsifier agent is added to the system).

To validate this approach, in the next step, we have taken a close look at the scientific literature in the search for works that have systematically investigated the stability of crude oil emulsions having either different formulations (change in salinity or addition of demusifiers) or different temperatures while taking great care to characterize the IFT of their systems. From these investigations, we have found several relevant articles dealing with various $\mathrm{W} / \mathrm{O}$ emulsion systems, which we present and discuss here. Using bottle test experiments, Bouriat et al. ${ }^{39}$ have screened the influence of several model demulsifiers, including Tween 80 , NP15EO, and triblock pluronic series, on the stability of $\mathrm{W} / \mathrm{O}$ crude oil emulsions diluted in cyclohexane. 
Using a similar experimental approach, Li et al. ${ }^{40}$ have tested the efficiency of synthetic demulsifiers based on natural tanic acid to phase separate crude oil emulsions. Alsabagh et al. ${ }^{41}$ and Kang et $\mathrm{al}^{42}$ have investigated the stability loss of crude oil emulsions caused by the addition of a demulsifier agent based on polyethylene and propylene oxides. El-Sharaky et al. $^{43}$ have performed research on new star polymeric nonionic surfactants as crude oil emulsion breakers. Wen et al. ${ }^{44}$ have analyzed the biological demulsification process of a W/O emulsion. For all of these aforementioned works, we have carefully read the mixing operating conditions in play during the emulsification process while taking good note of the values of the physical quantities (water-oil surface tension, oil viscosity, and oil density) characterizing the systems investigated. From these gathered data, we can estimate the Reynolds numbers in play during the emulsification process and conclude that for all of these reported studies the formation of droplets which occurs under turbulent flow falls into the inertial subrange regime.

By using eq 12 with $n=3 / 5$ and the reported IFT measurements, we first compute the predicted value for $\tau_{\mathrm{cB}} /$ $\tau_{\mathrm{cA}}$, the relative lifetime of the two studied emulsions (the reference one, $\mathrm{A}$, and the other one, $\mathrm{B}$ ). We underline the fact that the two emulsions, $\mathrm{A}$ and $\mathrm{B}$, that are studied and whose lifetimes are compared in each article (i) possess the same aqueous and oil phases and (ii) are prepared using the same emulsification protocol. However, their surfactant monolayers exhibit different spontaneous curvatures caused either by the addition of a demulsifier in emulsion $\mathrm{B}$ or by a change in the salinity of the aqueous phase. For all of these articles, we also extract the experimental values of $\tau_{\mathrm{cB}} / \tau_{\mathrm{cA}}$ that we next compare to the previously predicted values, as depicted in Figure 9. (See

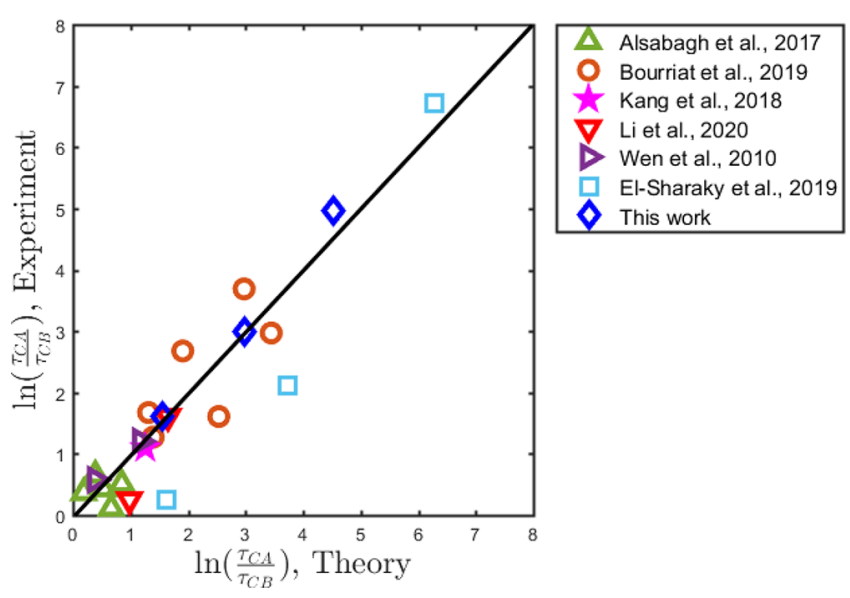

Figure 9. Comparison between theoretical predictions from our model and experimental data obtained for different water-in-crude oil emulsion systems. The predicted values have been found by taking $\kappa$ $=0.59 k_{\mathrm{B}} T, \bar{\kappa}=-0.58 k_{\mathrm{B}} T$, and $b_{0}=1.7 \mathrm{~nm}$ for all investigated systems.

the SI for more information.) Strikingly, we observe that all experimental data collapse remarkably well onto the theoretical prediction given by eq 12 , indicating that the stability behavior of these W/O systems seems to be universal. Our results reveal the very good correlation that exists between the relative stability of two W/O emulsion systems and their respective IFT values. Of course, it is important to point out that the universal behavior predicted by eq 12 and experimentally evidenced in Figure 9 stands only because we assume that the values of $\kappa$ and $\bar{\kappa}$ are similar for all emulsion systems as remarked on in the previous section and because we have not taken into account the complexity of the crude oil systems. Although the theoretical predictions follow well the trend of the experimental points as shown in Figure 9, they are not in completely good agreement. This can be explained by the variation of $b_{0}, \kappa$, and $\bar{\kappa}$ of different formulations.

From the very good agreement found in Figure 9 between experimental findings and theoretical predictions, we conclude that our crude model quantitatively well describes the stability of emulsions with IFT values in the range of $10^{-3}-10^{2} \mathrm{mN} / \mathrm{m}$. In addition, our results indicate that the bending and saddle-splay moduli $\kappa$ and $\bar{\kappa}$ of the surfactant monolayers that naturally favor the formation of $\mathrm{W} / \mathrm{O}$ crude oil systems likely take values of around $\kappa=0.59 k_{\mathrm{B}} T$ and $\bar{\kappa}=-0.58 k_{\mathrm{B}} T$. Very importantly, our experimental findings and theoretical predictions unveil the crucial importance of reducing the IFT of a W/O system to efficiently improve its destabilization, as already noticed by several authors.. ${ }^{32,45}$

\section{CONCLUSIONS}

We have studied the stability loss of Span 80-stabilized W/O emulsions caused by the addition of Tween 20, a water-soluble surfactant that acts as a demulsifier and promotes coalescence in $\mathrm{W} / \mathrm{O}$ emulsion systems. Following the experimental methodology recently introduced by Dinh et al., ${ }^{11}$ we have extracted the values of $\omega$, the coalescence rate per unit area, for these systems through systematic bottle test experiments; the use of dropdrop coalescence experiments has corroborated the results obtained in the bottle tests. Our experimental findings show that the lifetime of the W/O emulsion significantly decreases with the amount of Tween 20 added to it. We have rationalized our experimental results by introducing a microscopic model that explains the effect of a surfactant demulsifier added to a $\mathrm{W} / \mathrm{O}$ system on the occurrence of nucleating a hole through the oil interstitial film separating two adjacent water droplets. Within this theoretical framework, we have established an analytical formula that predicts that the ratio between the coalescence rates per unit are of two emulsions solely from the values of their respective IFTs that very well describe our experimental data. In addition, as already noticed by several authors, our results unveil the importance of reducing the IFT of an emulsion system in order to destabilize it. Despite its simplicity and the strong assumptions made, this analytical formula also remarkably well captures experimental findings obtained for a wide range of water-in-crude oil emulsion systems by other research groups. We anticipate that our results could be of great interest for the scientific community working in the field of emulsion science.

\section{APPENDIX A: ESTIMATION OF THE SURFACE OF CONTACT IN DROP-DROP EXPERIMENTS}

The mean coalescence time between two coalescing drops strongly depends on their surface of contact, $S_{c}$. We present here a simple image analysis method to estimate $S_{\mathrm{c}}$ accurately in a drop-drop experiment, as depicted in Figure 10. Note that our method works properly only when the surface of contact is very small but is difficult to estimate otherwise. Below, we detail the method. We assume that the surface of contact is a flat disk with radius $R_{\mathrm{c}}$.

Let us first consider the lower drop before it contacts the upper one. At its apex, the internal and external pressures, noted as $P_{\mathrm{i}}$ and $P_{\mathrm{e}}$, respectively, are related to each other by Laplace's law 


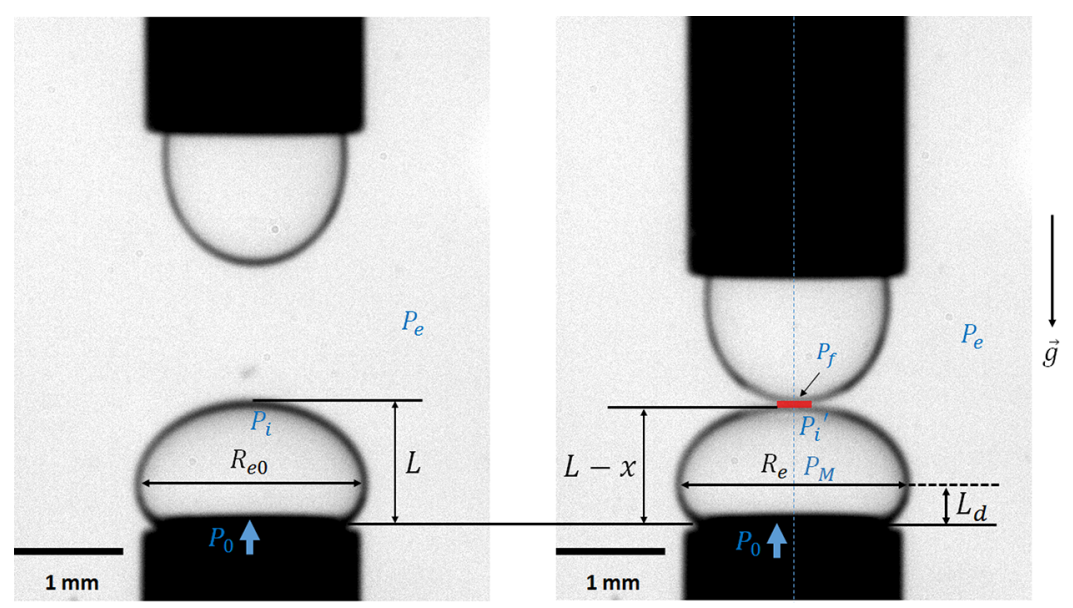

Figure 10. Side views of the two drops taken before and after contact. Shown are the various geometrical lengths defined in the text that permit us to easily estimate the surface of contact between the two drops by using eq 19 .

$$
P_{\mathrm{i}}-P_{\mathrm{e}}=\frac{2 \gamma}{R_{0}}
$$

where $\gamma$ is the interfacial tension between the two immiscible phases and $R_{0}$ is the curvature radius at the apex. Let us define $P_{0}$ as the pressure applied to the liquid at the tip of the lower needle by the syringe pump to overcome the Laplace pressure and generate the water drop immersed in the continuous oil phase. The hydrostatic pressure felt inside the drop leads to

$$
P_{\mathrm{i}}+\Delta \rho g L=P_{0}
$$

where $\Delta \rho$ is the water/oil density difference and $L$ is the length between the apex of the drop and the tip of the needle as shown in Figure 10. $L_{\mathrm{d}}$ describes the length between the height position of the equator, where the radius of the drop is at its maximum, and the tip of the needle.

Now let us consider the two drops in contact, at rest before they merge. The extra pressure caused by the contact leads to a small deformation of the apex of the lower drop, $x$, and a small deformation of the equator line of the lower droplet that we note as $\Delta R_{\mathrm{e}}=R_{\mathrm{e}}-R_{\mathrm{e} 0}$. Note that $x \ll L$ and $\Delta R_{\mathrm{e}} \ll R_{\mathrm{e} 0}$. This contact also creates a thin oil film between the two drops in which the pressure is $P_{\mathrm{f}}$. If one assumes that this film is flat, then the internal liquid pressure at the apex of the lower drop is $P_{\mathrm{f}}=P_{\mathrm{i}}{ }^{\prime}$. Writing the hydrostatic pressure profile inside the deformed drop yields

$$
P_{\mathrm{f}}+\Delta \rho g(L-x)=P_{0}
$$

The water internal pressure at the equator line, $P_{\mathrm{M}}$, is given by $P_{\mathrm{M}}=P_{0}-\Delta \rho g L_{\mathrm{D}}$, where $L_{\mathrm{D}}$ is the distance between the equator and the needle. Note that although the lower drop deforms because of its contact with the upper one, the length $L_{\mathrm{d}}$ remains mostly constant since the droplet volume is unchanged due to its incompressibility.

We next analyze the force balance that applies to the part of the lower drop located above its equator line after contact with the upper drop is made. The vertically upward component of the exerted force on this part of the drop which is induced by the pressure $P_{\mathrm{M}}$ felt at the equator line can be expressed as $F_{\text {up }}=$ $P_{\mathrm{M}} \pi R_{\mathrm{e}}{ }^{2}=\left(P_{0}-\Delta \rho g L_{\mathrm{D}}\right) \pi R_{\mathrm{e}}{ }^{2}$, where $R_{\mathrm{e}}$ is the new radius of the equator line. The vertical component of the downward force $F_{\text {down }}$ exerted on this drop includes the sum of the gravitational and buoyancy forces $\Delta \rho g V_{\text {up }}$ where $V_{\text {up }}$ corresponds to the volume of the part of the drop that is located above its equator line, the capillary force $2 \pi R_{\mathrm{e}} \gamma$, the force induced by the exterior pressure $P_{\mathrm{e}} \pi\left(R_{\mathrm{e}}^{2}-R_{\mathrm{c}}^{2}\right)$, and the force exerted by the pressure inside the thin oil film $\mathrm{P}_{f} \pi R_{c}^{2}$. By summing these terms, one obtains the force balance equation applied to this part of the lower drop:

$$
\left(P_{0}-\Delta \rho g L_{\mathrm{D}}\right) \pi R_{\mathrm{e}}{ }^{2}=\Delta \rho g V_{\text {up }}+2 \pi R_{\mathrm{e}} \gamma+P_{\mathrm{e}} \pi\left(R_{\mathrm{e}}{ }^{2}-R_{\mathrm{c}}{ }^{2}\right)+P_{\mathrm{f}} \pi R_{\mathrm{c}}{ }^{2}
$$

By combining eqs 14-17, one can derive a relationship among $R_{\mathcal{c}}$, and $R_{\mathrm{e}}, V_{\text {up }}, L_{\mathrm{D}}$, and $x$ :

$$
\begin{aligned}
& {\left[\frac{2 \gamma}{R_{0}}+\Delta \rho g\left(L-L_{\mathrm{D}}\right)\right] \pi R_{\mathrm{e}}^{2}} \\
& =\Delta \rho g V_{\text {up }}+2 \pi R_{\mathrm{e}} \gamma+\left(\frac{2 \gamma}{R_{0}}+\Delta \rho g x\right) \pi R_{\mathrm{c}}{ }^{2}
\end{aligned}
$$

When the deformation is small, a first-order expansion in terms of $\Delta R_{\mathrm{e}}$ of this relationship leads to

$$
\left[\frac{2 \gamma}{R_{0}}+\Delta \rho g\left(L-L_{\mathrm{D}}\right)\right] 2 \pi R_{\mathrm{e} 0} \Delta R_{\mathrm{e}} \simeq 2 \pi \Delta R_{\mathrm{e}} \gamma+\left(\frac{2 \gamma}{R_{0}}+\Delta \rho g x\right) \pi R_{\mathrm{c}}{ }^{2}
$$

Equation 19 enables us to simply estimate the radius of contact $R_{\mathrm{c}}$ from the known physical-chemical parameters of our system, $\gamma=5.2 \mathrm{mN} / \mathrm{m}$ and $\Delta \rho=250 \mathrm{~kg} / \mathrm{m}^{3}$, and from some geometrical parameters that can be measured using an image analysis of two side views of the drop taken before and after contact with the other drop. For example, from an image analysis of Figure 10 performed with Image J, we measured $R_{\mathrm{e} 0}=1.06 \pm$ $0.005 \mathrm{~mm}, \Delta R_{\mathrm{e}}=0.02 \pm 0.005 \mathrm{~mm}, L=1.12 \pm 0.005 \mathrm{~mm}, L_{\mathrm{D}}=$ $0.4 \pm 0.005 \mathrm{~mm}$, and $x=0.08 \pm 0.005 \mathrm{~mm}$. By substituting these collected data values into eq 19 , we can then estimate that $R_{\mathrm{c}}=$ $0.17 \pm 0.02 \mathrm{~mm}$ and that the surface of contact between the two drops is roughly $0.1 \pm 0.01 \mathrm{~mm}^{2}$.

In addition, we have also performed another experiment where the contact surface area is increased while keeping the same formulation ( $800 \mathrm{ppm}$ Span 80 in dodecane) and droplet volume with the above experiment, as shown in Figure 11. Applying the above method, we found that the estimated surface of contact in this case is about $0.4 \pm 0.01 \mathrm{~mm}^{2}$. 


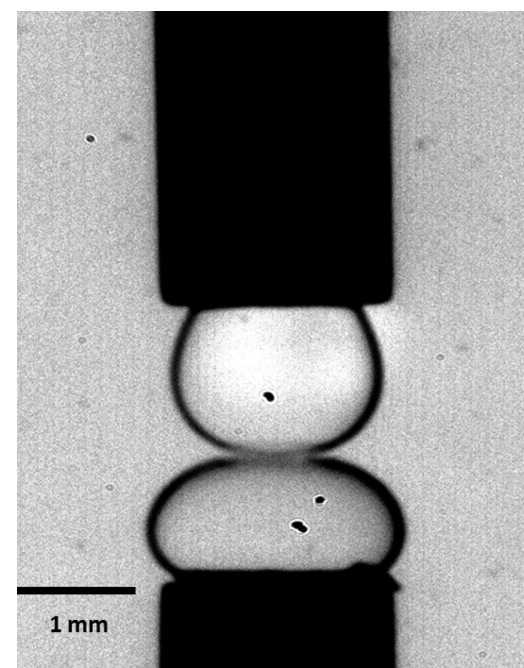

Figure 11. Side view of drop-drop experiment at higher surface contact $S_{\mathrm{c}}$

\section{APPENDIX B: APPROXIMATE ANALYTICAL EXPRESSION OF $W^{\star}$}

Here, we derive an analytical expression that well approximates $W^{\star}$. First, we express $W\left(a, b_{0}\right)$ in terms of the dimensionless variable $u=1+a / b_{0}$ :

$$
\begin{gathered}
\frac{W(u)}{2 \pi \kappa}=\frac{b_{0}^{2}}{\lambda^{2}}\left(\pi u-u^{2}-2\right)-2 K_{0} b_{0}(\pi u-4) \\
+\frac{2 u^{2}}{\sqrt{u^{2}-1}} a \tan \sqrt{\frac{u+1}{u-1}}-4-\frac{2 \bar{\kappa}}{\kappa}
\end{gathered}
$$

Second, by plotting $\frac{W(u)}{2 \pi \kappa}$ versus $u$, we notice that the maximum value, $\frac{W^{\star}}{2 \pi \kappa}$, is found for a value of $u^{\star}$ for which the function $g(u)=\frac{2 u^{2}}{\sqrt{u^{2}-1}} a \tan \sqrt{\frac{u+1}{u-1}}$ is well approximated by its linear asymptote $g(u) \underset{u \rightarrow \infty}{\longrightarrow} 2+\frac{\pi}{2} u$. Therefore, by replacing $g(u)$ in eq 20 with its asymptotic expansion $2+\frac{\pi}{2} u$, we can determine an approximate value of $W(u)$ and subsequently derive an analytical expression for the maximum in $W(u)$, as shown below:

$$
\frac{W^{\star}}{2 \pi \kappa} \simeq \pi^{2} \frac{\left[\left(\frac{b_{0}{ }^{2}}{\lambda^{2}}-2 K_{0} b_{0}\right)+\frac{1}{2}\right]^{2}}{\frac{4 b_{0}{ }^{2}}{\lambda^{2}}}-\frac{2 b_{0}{ }^{2}}{\lambda^{2}}+8 K_{0} b_{0}-2-\frac{2 \bar{\kappa}}{\kappa}
$$

\section{ASSOCIATED CONTENT}

\section{(5) Supporting Information}

The Supporting Information is available free of charge at https://pubs.acs.org/doi/10.1021/acs.langmuir.1c00975.

Equilibrium interfacial tension measurements of different concentrations of Span 80 or Tween 20 and of surfactant mixtures; dynamical interfacial tension measurements used to estimate the bulk diffusion coefficient; and details of experimental findings from other papers given in Figure 9 (PDF)

\section{AUTHOR INFORMATION}

\section{Corresponding Author}

Huy-Hong-Quan Dinh - Laboratoire Physico-Chimie des Interfaces Complexes, 64170 Lacq, France; TOTAL S.A., Pôle d'Etudes et de Recherches de Lacq, 64170 Lacq, France; (1) orcid.org/0000-0002-2353-3219; Email: huy-hongquan.dinh@total.com

\section{Authors}

Enric Santanach-Carreras - Laboratoire Physico-Chimie des Interfaces Complexes, 64170 Lacq, France; TOTAL S.A., Pôle d'Etudes et de Recherches de Lacq, 64170 Lacq, France

Marie Lalanne-Aulet - Laboratoire Physico-Chimie des Interfaces Complexes, 64170 Lacq, France

Véronique Schmitt - Centre de Recherche Paul Pascal, 33600 Pessac, France; 이이이.org/0000-0002-9938-9911

Pascal Panizza - IPR, UMR CNRS 6251, Campus Beaulieu, Université Rennes 1, 35042 Rennes, France; Laboratoire Sciences et Ingénierie de la Matière Molle, ESPCI Paris, PSL University, Sorbonne Université, CNRS UMR 7615, 75005 Paris, France

François Lequeux - Laboratoire Sciences et Ingénierie de la Matiere Molle, ESPCI Paris, PSL University, Sorbonne Université, CNRS UMR 7615, 75005 Paris, France; (1) orcid.org/0000-0003-4076-3988

Complete contact information is available at: https://pubs.acs.org/10.1021/acs.langmuir.1c00975

\section{Notes}

The authors declare no competing financial interest.

\section{ACKNOWLEDGMENTS}

This work was funded by a French CIFRE fellowship (contract number 2018-0911) with Total S.A. We acknowledge Total S.A. management for giving us permission to publish our results. We thank Maurice Bourrel, Nicolas Passade-Boupat, and Thierry Palermo for many fruitful discussions. We also thank Fatoumata Coulibaly and Franco Mpandi for helping us with the dropdrop experiment and Didier Lauranson for his support in daily laboratory activities.

\section{REFERENCES}

(1) Leal-Calderon, F.; Schmitt, V.; Bibette, J. Emulsion Science: Basic Principles; Springer: New York, 2007.

(2) Langevin, D. Bubble coalescence in pure liquids and in surfactant solutions. Curr. Opin. Colloid Interface Sci. 2015, 20 (2), 92-97.

(3) de Vries, A. J. Foam stability: Part V. Mechanism of film rupture. J. Recl. Trav. Chim. Pays-Bas 1958, 77 (5), 441-461.

(4) de Gennes, P.-G. Some remarks on coalescence in emulsions or foams. Chem. Eng. Sci. 2001, 56 (19), 5449-5450.

(5) Calvo, E.; de Malmazet, E.; Risso, F.; Masbernat, O. Coalescence of Water Drops at an Oil-Water Interface Loaded with Microparticles and Surfactants. Ind. Eng. Chem. Res. 2019, 58 (34), 15573-15587.

(6) Schmitt, V.; Leal-Calderon, F. Measurement of the coalescence frequency in surfactant-stabilized concentrated emulsions. Europhys. Lett. 2004, 67 (4), 662-668.

(7) Hasmy, A.; Paredes, R.; Sonneville-Aubrun, O.; Cabane, B.; Botet, R. Dynamical Transition in a Model for Dry Foams. Phys. Rev. Lett. 1999, 82 (16), 3368.

(8) Rekvig, L.; Frenkel, D. Molecular simulations of droplet coalescence in oil/water/surfactant systems. J. Chem. Phys. 2007, 127 (13), 134701. 
(9) Deminiere, B.; Colin, A.; Leal-Calderon, F.; Muzy, J. F.; Bibette, J. Cell Growth in a 3D Cellular System Undergoing Coalescence. Phys. Rev. Lett. 1999, 82 (1), 229.

(10) Forel, E.; Dollet, B.; Langevin, D.; Rio, E. Coalescence in TwoDimensional Foams: A Purely Statistical Process Dependent on Film Area. Phys. Rev. Lett. 2019, 122 (8), 088002.

(11) Dinh, H.-H.-Q.; Santanach-Carreras, E.; Schmitt, V.; Lequeux, F. Coalescence in concentrated emulsions: Theoretical predictions and comparison with experimental bottle test behaviour. Soft Matter 2020, 16 (45), 10301-10309.

(12) Kabalnov, A.; Wennerstrom, H. Macroemulsion Stability: The Oriented Wedge Theory Revisited. Langmuir 1996, 12 (2), 276-292.

(13) Kabalnov, A.; Weers, J. Macroemulsion Stability within the Winsor III Region: Theory versus Experiment. Langmuir 1996, 12 (8), 1931-1935.

(14) Roger, K.; Cabane, B. Why Are Hydrophobic/Water Interfaces Negatively Charged ? Angew. Chem., Int. Ed. 2012, 51 (23), 5625-5628.

(15) Ghosh, P.; Juvekar, V.A. Analysis of the drop rest phenomenon. Chem. Eng. Res. Des. 2002, 80 (7), 715-728.

(16) Tanford, C. The Hydrophobic Effect: Formation of Micelles and Biological Membranes, 2nd ed.; Wiley: New York, 1980.

(17) Israelachvili, J. N.; Mitchell, D. J.; Ninham, B. W. Theory of selfassembly of hydrocarbon amphiphiles into micelles and bilayers. $J$. Chem. Soc., Faraday Trans. 2 1976, 72, 1525-1568.

(18) Wang, H.; Davies, R. H. Simultaneous sedimentation and coalescence of a dilute dispersion of small drops. J. Fluid Mech. 1995, 295, 247-261.

(19) Rupprecht, J.-F.; Ong, K. H.; Yin, J.; Huang, A.; Dinh, H.-H.-Q.; Singh, A. P.; Zhang, S.; Yu, W.; Saunders, T. E. Geometric constraints alter cell arrangements within curved epithelial tissues. Mol. Biol. Cell 2017, 28 (25), 3582-3594.

(20) Kashchiev, D.; Exerowa, D. Nucleation Mechanism of Rupture of Newtonian Black Films. I. Theory. J. Colloid Interface Sci. 1980, 77 (2), 501-511.

(21) de Vries, A. Foam stability: Part V. Mechanism of film rupture. Rec. Trav. Chim. 1958, 77 (5), 441-461.

(22) Würger, A. Bending Elasticity of Surfactant Films: The Role of the Hydrophobic Tails. Phys. Rev. Lett. 2000, 85 (2), 337-341.

(23) Leitao, H.; Somoza, A. M.; da Gama, M. M. T. Scaling of the interfacial tension of microemulsions: A phenomenological description. J. Chem. Phys. 1996, 105 (7), 2875-2883.

(24) Politova, N.; Tcholakova, S.; Denkov, N. D. Factors affecting the stability of water-oil-water emulsion films. Colloids Surf., A 2017, 522, 608-620.

(25) Binks, B.; Meunier, J.; Abillon, O.; Langevin, D. Mesurement of film rigidity and interfacial-tensions in several ionic surfactant oil-water microemulsion systems. Langmuir 1989, 5 (2), 415-421.

(26) Safran, S. Saddle-splay modulus and the stability of spherical microemulsion. Phys. Rev. A: At., Mol., Opt. Phys. 1991, 43 (6), 2903.

(27) Khademi, M.; Sham, S.; Cheng, Y.; Barz, D. P. J. Charge and Electrical Double Layer Formation in a Nonpolar Solvent Using a Nonionic Surfactant. Langmuir 2020, 36 (19), 5156-5164.

(28) Abou-Nemeh, I.; Bart, H. J. Microstructures in the System Water/D2EHPA/Span-80/n-Dodecane. Langmuir 1998, 14 (16), 4451-4459.

(29) Campanelli, J. R.; Wang, X. Dynamic Interfacial Tension of Surfactant Mixtures at Liquid-Liquid Interfaces. J. Colloid Interface Sci. 1999, 213 (2), 340-351.

(30) Politova, N. I.; Tcholakova, S.; Tsibranska, S.; Denkov, N. D.; Muelheims, K. Coalescence stability of water-in-oil drops: Effects of drop size and surfactant concentration. Colloids Surf., A 2017, 531, 3239.

(31) Narayan, S.; Makhnenko, I.; Moravec, D.; Hauser, B.; Dallas, A.; Dutcher, C. S. Insights into the Microscale Coalescence Behavior of Surfactant-Stabilized Droplets Using a Microfluidic Hydrodynamic Trap. Langmuir 2020, 36 (33), 9827-9842.

(32) Marquez, R.; Forgiarini, A. M.; Langevin, D.; Salager, J.-L. Instability of Emulsions Made with Surfactant-Oil-Water Systems at
Optimum Formulation with Ultralow Interfacial Tension. Langmuir 2018, 34 (31), 9252-9263.

(33) Kolmogorov, A. A refinement of previous hypotheses concerning the local structure of turbulence in a viscous incompressible fluid at high Reynolds numbers. J. Fluid Mech. 1962, 13 (1), 82-85.

(34) Boxall, J. A.; Koh, C. A.; Sloan, E. D.; Sum, A. K.; Wu, D. T. Droplet Size Scaling of Water-in-Oil Emulsions under Turbulent Flow. Langmuir 2012, 28 (1), 104-110.

(35) Vankova, N.; Tcholakova, S.; Denkov, N.; Ivanov, I. B.; Vulchev, V. D.; Danner, T. Emulsification in turbulent flow: 1. Mean and maximum drop diameters in inertial and viscous regimes. J. Colloid Interface Sci. 2007, 312 (2), 363-380.

(36) Kolmogorov, A. On the breakage of drops in a turbulent flow. Dokl. Akad. Nauk. SSSR 1949, 66, 825-828.

(37) Taylor, G. I. The formation of emulsions in definable field of flows. Proc. R. Soc. London Ser. A 1934, 146 (858), 501-523.

(38) Mason, T. G.; Bibette, J. Shear rupturing of droplets in complex fluids. Langmuir 1997, 13 (17), 4600-4613.

(39) Bouriat, P.; Rondon, M.; Lachaise, J.; Salager, J.-L. Correlation between Interfacial Tension Bump and Optimal Crude Oil Dehydration. Energy Fuels 2009, 23 (8), 3998-4002.

(40) Li, Z.; Geng, H.; Wang, X.; Jing, B.; Liu, Y.; Tanb, Y. Noval tannic acid-based polyether as an effective demulsifier for water-in-aging crude oil emulsions. Chem. Eng. J. 2018, 354, 1110-1119.

(41) Al-Sabagh, A. M.; Elsharaky, E. A.; El-Tabey, A. E. Demulsification Performance and the Relative Solubility Number (RSN) of Modified Poly(maleic anhydride-alt-1-dodecene) on Naturally Asphaltenic Crude Oil Emulsion. J. Dispersion Sci. Technol. 2017, 38 (2), 288-295.

(42) Kang, W.; Yin, X.; Yang, H.; Zhao, Y.; Huang, Z.; Hou, X.; Sarsenbekuly, B.; Zhu, Z.; Wang, P.; Zhang, X.; Geng, J.; Aidarova, S. Demulsification performance, behavior and mechanism of different demulsifiers on the light crude oil emulsions. Colloids Surf., A 2018, 545, 197-204.

(43) El-Sharaky, E.-S. A.; El-Tabey, A. E.; Mishrif, M. R. Novel Star Polymeric Nonionic Surfactants as Crude Oil Emulsion Breakers. J. Surfactants Deterg. 2019, 22, 779-793.

(44) Wen, Y.; Cheng, H.; Lu, L.-J.; Liu, J.; Feng, Y.; Guan, W.; Zhou, Q.; Huang, X.-F. Analysis of biological demulsification process of waterin-oil emulsionbyAlcaligenessp. S-XJ-1. Bioresour. Technol. 2010, 101, $8315-8322$

(45) Marquez, R.; Forgiarini, A. M.; Langevin, D.; Salager, J.-L. Breaking of Water-In-Crude Oil Emulsions. Part 9. New Interfacial Rheology Characteristics Measured Using a Spinning Drop Rheometer at Optimum Formulation. Energy Fuels 2019, 33 (9), 8151-8164. 